\title{
Categorising visual hallucinations in early Parkinson's disease
}

Benjamin J. Clegg*a, Gordon W.Duncan ${ }^{a, b}$, Tien K. Khooc, d, Roger A. Barkere, David J.

Burn $^{f}$, Alison J. Yarnall ${ }^{\mathrm{a}, \mathrm{g}}$ Rachael A. Lawson ${ }^{\mathrm{a}, \mathrm{g}}$

${ }^{a}$ Institute of Neuroscience, Newcastle University, UK,

${ }^{b}$ Centre for Clinical Brain Sciences, University of Edinburgh, UK.

c School of Medicine and Menzies Health Institute Queensland, Griffith University, Australia

${ }^{\mathrm{d}}$ School of Medicine, University of Wollongong, New South Wales, Australia

e John Van Geest Centre for Brain Repair, University of Cambridge, UK

${ }^{f}$ Faculty of Medical Science, Newcastle University, UK

${ }^{\mathrm{g}}$ Newcastle University Institute for Aging, Newcastle University, UK,

*Corresponding author - Benjamin Clegg, b.clegg@newcastle.ac.uk, Newcastle Magnetic

Resonance Centre, Campus for Ageing and Vitality, Newcastle University, Newcastle upon

Tyne, NE4 5PL. 01912086000

Running Title: Visual hallucinations in Parkinson's disease

Key Words: Parkinson's disease, Visual hallucinations, Cognition, Quality of life 


\section{Abstract}

\section{Background}

Visual hallucinations (VHs) are common in Parkinson's disease (PD), with prevalence ranging from 27-50\% in cross-sectional cohorts of patients with well established disease. However, minor hallucinations may occur earlier in the disease process than previously reported and so we sought to categorise VHs in a cohort of newly diagnosed PD patients and their relationship to other clinical features.

\section{Methods}

Newly diagnosed PD participants ( $n=154)$ were recruited as part of the Incidence of Cognitive Impairment in Cohorts with Longitudinal Evaluation in PD (ICICLE-PD) study. Participants completed the Movement Disorders Society Unified Parkinson's Disease Rating Scale (MDS-UPDRS III), Montreal Cognitive Assessment (MoCA), and Parkinson's Disease Questionnaire (PDQ-39) to assess motor severity, cognition and quality of life (QoL), respectively. VHs were classified using the North East Visual Hallucinations Inventory. Hierarchical regression was used to build predictive models of motor severity, QoL and cognition.

\section{Results}

$22 \%(n=34)$ of participants experienced recurrent VHs with minor VHs being most frequently reported ( $64.7 \%$ of hallucinators). Complex VHs were present in $32.4 \%$ of hallucinating participants. Linear regression showed VHs predicted poorer PDQ-39 and MoCA scores $(\beta=0.201, p=0.006$ and $\beta=-$ $0.167, p=0.01$, respectively) but not motor severity ( $p>0.05)$.

\section{Conclusions}


Over a fifth of people with newly diagnosed PD reported recurrent VHs; minor hallucinations were the most common, although a small proportion reported complex VHs. Recurrent VHs were found to be a significant independent predictor of cognitive function and QoL but not motor severity. Our findings highlight the importance of screening for VHs at diagnosis.

\section{Introduction}

Visual hallucinations (VHs) are common in Parkinson's disease (PD) with prevalence rates ranging from $27-50 \%$ in patients with well-established disease [1]. Their presence can be distressing and has been associated with poorer quality of life (QoL) in both patients [2] and caregivers [3] as well as cognitive impairment [4] with progression to dementia (PDD) and motor severity $[1,8][5]$. Risk factors associated with VHs in PD are disease severity and depression $[1,8,9]$, although this association appears to be less pronounced in early PD participants with some such studies finding no association at all $[10,11]$.

Fully formed complex VHs are usually seen at later stages of PD and in PDD [12]. In contrast minor hallucinations, which have previously been defined as a feeling of presence or passing of an entity [1] and typically last a few seconds, have been said to occur in as many as $42 \%$ of PD patients, including those in early stage disease [11]. Furthermore, minor VHs have been found to be less malignant that complex VHs in terms of associated risk of cognitive impairment [13], but this is unresolved and may be a more significant predictor of cognitive problems in patients in early stage disease. We therefore sought to address this question of the characteristics of hallucinations in newly diagnosed incident PD and their relationship to motor severity, cognitive function and QoL. 


\section{Methods}

\section{Participants}

Subjects participated in the Incidence of Cognitive Impairment in Cohorts with Longitudinal Evaluation in PD (ICICLE-PD) study [15]. Newly diagnosed idiopathic PD patients from Newcastle upon Tyne and Gateshead (UK) were invited to participate between June 2009 and December 2011 from community and outpatient clinics. Patients were diagnosed by a movement disorder specialist and met Queen's Square Brain Bank criteria for idiopathic PD [16]. Patients were excluded if they had significant cognitive impairment defined as a Mini Mental State Examination (MMSE) score $<24$ or a diagnosis of dementia [17]. Patients with insufficient English to be assessed, PD diagnosis before the onset of the study, drug-induced parkinsonism, vascular parkinsonism and atypical forms of parkinsonism such as progressive supranuclear palsy, multiple system atrophy, or corticobasal degeneration were also excluded. This study was approved by the Newcastle and North Tyneside Research Ethics Committee and performed according to the Declaration of Helsinki. All subjects provided written informed consent.

\section{Assessments}

Participants completed a schedule of assessments. Participants were assessed when "on" their normal anti-PD medication. Demographic data included age, gender, disease duration and number of years in education. PD medications were recorded and levodopa equivalent daily dose (LEDD) was calculated [18]. Disease severity was assessed using the Movement Disorders Society Unified Parkinson's Disease Rating Scale (MDS-UPDRS) part II and III [19] and Hoehn and Yahr stage [20]. Motor phenotypes were defined using the MDS-UPDRS scale as Postural Instability/Gait difficulty (PIGD), Tremor Dominant (TD) or Indeterminate phenotype [21].

The Geriatric Depression Scale (GDS-15) [22] was used to assess depression. Excessive daytime sleepiness was assessed using the Epworth Sleepiness Scale (ESS) [23]; the Pittsburgh Sleep Quality 
Index (PSQI) [24] was used to assess sleep quality and disturbance. Quality of life was assessed using the Parkinson's Disease Questionnaire (PDQ-39, with higher scores representing poorer QoL) [25].

The MMSE [26] and Montreal Cognitive Assessment (MoCA) [27] were used to assess global cognition. We used modified PD-MCl criteria to determine whether a patient had $\mathrm{MCl}$ - namely scores that were 1.5 standard deviations (SD) below normative means $[15,28]$ in two tests, either within one cognitive domain or in two different domains, as described previously [29].

An assessment of VHs was conducted using the North East Visual Hallucination Inventory (NEVHI) [30]. This semi-structured interview identifies and assesses VHs by category (complex, illusions, simple and minor (Supplementary Table 1)), and by frequency. For the purposes of this study, floaters and hallucinations occurring in the context of a migraine were excluded and analyses focused on recurrent hallucinations in the last month. Recurrent was defined as more than one separate episode of hallucination, as per the NEVHI. This criteria was used to classify VHs in the analysis to exclude those who had had hallucinations in a non PD context such as, for example, from an episode of delirium.

\section{Statistical analysis}

Statistical analyses were performed using SPSS (Version 21.0. Armonk, NY: IBM Corp). The normality distribution of the data was assessed using the Kolmogorov-Smirnov test and visual histograms. Differences between two groups were assessed using either independent t-tests or Mann-Whitney $\mathrm{U}$ tests as appropriate. Comparisons between more than two groups were conducted with KruskalWallis tests. Chi-squared tests were used to compare differences between categorical variables. Significance was set at $p<0.05$ for all analyses. Bonferroni's correction was applied for multiple comparisons.

Hierarchical regression was used to determine predictors of QoL, cognition and motor severity. Backwards stepwise regression was used and non-significant predictors were excluded to produce a 
basic model for each dependent variable. Predictors of PDQ-39 scores included GDS-15 scores, gender, years of education, age and MDS-UPDRS part III scores [31, 32]. Predictors of cognition using MoCA scores included in the initial model comprised ESS scores, MDS-UPDRS part III scores, age and years of education [15]. Predictors of MDS-UPDRS part III scores included in the initial model included gender, GDS-15 scores, PD-MCl and age [33]. To determine whether VHs were an independent predictor of each outcome, occurrence of recurrent VHs or not was subsequently added as a binary variable to the basic model.

\section{Results}

Of the 158 individuals enrolled [34], 154 participants completed the NEVHI and were therefore included in this study (Table 1). Application of the NEVHI identified $38.3 \%(n=59)$ of the participants as having any previous VHs. Of these, $22.1 \%(n=34)$ reported having recurrent VHs in the last month. Of the participants reporting recurrent $\mathrm{VHs}, 41.2 \%(n=14)$ reported more than one type of $\mathrm{VH}$. The most frequently reported classification of $\mathrm{VHs}$ were minor hallucinations $(64.7 \%, \mathrm{n}=22), 38.2 \%$ ( $n=13)$ of participants with recurrent VHs reported recurrent simple VHs and $20.6 \%(n=7)$ recurrent illusions. Cumulatively, 11 participants (32.4\%) reported recurrent complex VHs comprising of animals and people, including familiar and deceased individuals.

\section{Clinical features of patients with and without VHs}

The clinical characteristics of those patients with and without recurrent VHs were compared (Table 2). There was no difference in age, but those with recurrent VHs were more likely to be male. Participants with recurrent VHs had significantly poorer QoL, (PDQ-39 $26.7 \pm 17.0$ vs. $16.4 \pm 12.7$, respectively, $p=0.001$ ), higher daytime somnolence scores (ESS $8.8 \pm 5.0$ vs. $5.4 \pm 4.1$, respectively, $p<0.001$ ) and more depressive symptoms (GDS-15 $3.9 \pm 3.1$ vs. $2.5 \pm 2.3$, respectively, $p=0.015$ ) compared to those without VHs. Lower global cognition scores were found in participants with VHs compared to those without using both the MoCA and the MMSE scores(Table 2). 
A trend towards a difference in motor phenotypes was found between those with and without recurrent VHs (Table 2, $\mathrm{p}=0.063$ ). Within the recurrent $\mathrm{VH}$ group, there was a significantly higher proportion of participants identified as PIGD compared to indeterminate or tremor dominant phenotypes (61.8\% vs. $14.7 \%$ vs. $23.5 \%$, respectively; $\left.\chi^{2}=21.05, p<0.001\right)$.

Participants with recurrent VHs were categorised according to the most severe classification of hallucinations reported to create distinct groups of participants with VHs, such that complex VHs > illusions > simple VHs > minor VHs (Table 3). No significant differences in any clinical characteristic were found between groups. Post hoc analysis did not reveal significant differences when using two group comparisons after Bonferroni corrections were applied ( $p>0.008)$.

\section{The effects of $\mathrm{VH}$ on quality of life, cognition and motor severity}

We next investigated whether recurrent VHs were predictive of clinical features. Backwards stepwise linear regression was used to determine predictors of PDQ-39, MDS-UPDRS part III and MoCA scores. Occurrence of VHs were then added to the model. Covariates are listed in Table 4. Recurrent VHs were a significant predictor of poorer QoL scores, accounting for $2.3 \%$ of the variance $\left(\Delta R^{2}=0.023, p=0.006\right)$ and poorer cognition, accounting for $3.7 \%$ of the variance $\left(\Delta R^{2}=0.037\right.$, $p<0.001$, Table 4). However, recurrent VHs were not a significant predictor of MDS-UPDRS part III scores $(p>0.05)$.

\section{Discussion}

This study found recurrent VHs were reported in $22 \%$ of newly diagnosed PD participants. The most prevalent form was minor hallucinations, although $32 \%$ reported complex $\mathrm{VHs}$, and their presence was associated with both poorer cognition and QoL.

Our finding of 1 on 5 incident PD patients reporting VH is similar to that reported by Pagonabarraga et al. [11], where they found that $24 \%$ of drug-naïve early PD patients experienced VHs more than 
once per week, albeit in a smaller sample $(n=50)$. Our study also found, in line with this study of Pagonabarraga et al, that minor $\mathrm{VHs}$ were the most frequently reported $\mathrm{VH}$, occurring in $65 \%$ of recurrent hallucinators. However we also uniquely found that $32 \%$ of recurrent hallucinators reported complex VHs, which is surprising given their association with later stages of PD and PDD [4] and the fact that we found no differences in the clinical characteristics of patients with these different forms of VHs. This may have been due to the small numbers in each VH classification group.

However, we did find that recurrent VHs were significantly associated with worse global cognition scores using the MoCA, independent of age and years of education- although wat happens over time is still being investigated as we follow up this cohort.In particular, understanding the relationship over time between cognition and VHs could be useful in identifying those at risk of developing PDD in the future $[6,35]$.

Consistent with previous findings in newly diagnosed patients [11], there was no significant difference in motor phenotype classification or in motor severity and the presence or not of VHs. It has been previously reported that $\mathrm{VH}$ s are found in patients with greater motor severity of disease $[1,9]$, although this was generally in participants with more advanced PD than in our cohort.

We found that QoL was significantly poorer in participants reporting VHs, in line with earlier studies $[2,36]$. It has previously been suggested that reduced QoL in hallucinators may be due to depression [8] and reduced independence through nursing home placement [37]. However, our participants were newly diagnosed in early stages of PD, with none were living in nursing or residential care and only a few meeting a diagnostic criteria for depression, as measured by the GDS-15.

The main strengths of this study include a large well characterised representative incident cohort of patients, which by virtue of being a longitudinal study, means that misdiagnosis rates are minimised. The majority of participants in the present study were on dopaminergic medication (87.7\%) at the time of recruitment (even though the mean disease duration was only 6 months) which is 
representative of patients in real life clinical practice. Limitations included the small subgroup sizes, which limited statistical power to detect subtle differences between groups, as well as our analysis being cross-sectional, which means we cannot draw conclusions regarding the relationship between VHs and disease progression or cognitive decline. Finally, this study used global measures of cognition, which may have missed important findings regarding which specific cognitive modalities are associated with VHs early in PD.

In summary, we have found that VHs are common even in newly diagnosed PD patients, with minor hallucinations being the most frequently reported. Recurrent VHs in early PD were associated with poorer global cognition and QoL, but not with motor severity and as such may be an early harbinger of the development of PD dementia. However the longitudinal follow up of our cohort is needed to answer this question

\section{Conflicts of interest:}

The authors have no conflicts of interest to report.

\section{Funding sources:}

ICICLE-PD was funded by Parkinson's UK (J-0802, G-1301, G-1507). The research was supported by the Lockhart Parkinson's Disease Research Fund, the National Institute for Health Research (NIHR) Newcastle Biomedical Research Unit based at Newcastle upon Tyne Hospitals NHS Foundation Trust and Newcastle University and a NIHR Biomedical Research Centre award to the University of Cambridge/Addenbrooke's Hospital. 


\section{References}

[1] Fénelon G, Mahieux F, Huon R, Ziégler M (2000) Hallucinations in Parkinson's diseasePrevalence, phenomenology and risk factors. Brain 123, 733-745.

[2] Schrag A, Jahanshahi M, Quinn N (2000) What contributes to quality of life in patients with Parkinson's disease? Journal of Neurology, Neurosurgery \&amp; Psychiatry 69, 308-312.

[3] Aarsland D, Brønnick K, Ehrt U, De Deyn PP, Tekin S, Emre M, Cummings JL (2007) Neuropsychiatric symptoms in patients with Parkinson's disease and dementia: frequency, profile and associated care giver stress. Journal of Neurology, Neurosurgery \& Psychiatry 78, 36-42.

[4] Santangelo G, Trojano L, Vitale C, lanniciello M, Amboni M, Grossi D, Barone P (2007) A neuropsychological longitudinal study in Parkinson's patients with and without hallucinations. Mov Disord 22, 2418-2425.

[5] Hely MA, Reid WGJ, Adena MA, Halliday GM, Morris JGL (2008) The Sydney multicenter study of Parkinson's disease: the inevitability of dementia at 20 years. Movement disorders 23, 837-844.

[6] Janvin CC, Larsen JP, Aarsland D, Hugdahl K (2006) Subtypes of mild cognitive impairment in Parkinson's disease: progression to dementia. Movement Disorders 21, 1343-1349.

[7] Barnes J, David AS (2001) Visual hallucinations in Parkinson's disease: a review and phenomenological survey. Journal of Neurology, Neurosurgery \& Psychiatry 70, 727-733.

[8] Holroyd S, Currie L, Wooten GF (2001) Prospective study of hallucinations and delusions in Parkinson's disease. Journal of Neurology, Neurosurgery \& Psychiatry 70, 734-738.

[9] Lee AH, Weintraub D (2012) Psychosis in Parkinson's disease without dementia: Common and comorbid with other non-motor symptoms. Movement Disorders 27, 858-863.

[10] Aarsland D, Brønnick K, Alves G, Tysnes OB, Pedersen KF, Ehrt U, Larsen JP (2009) The spectrum of neuropsychiatric symptoms in patients with early untreated Parkinson's disease. Journal of Neurology, Neurosurgery \&amp; Psychiatry 80, 928-930.

[11] Pagonabarraga J, Martinez-Horta S, Fernandez de Bobadilla R, Perez J, Ribosa-Nogue R, Marin J, Pascual-Sedano B, Garcia C, Gironell A, Kulisevsky J (2016) Minor hallucinations occur in drug-naive Parkinson's disease patients, even from the premotor phase. Mov Disord 31, 45-52.

[12] Williams DR, Lees AJ (2005) Visual hallucinations in the diagnosis of idiopathic Parkinson's disease: a retrospective autopsy study. The Lancet Neurology 4, 605-610.

[13] Llebaria G, Pagonabarraga J, Martinez-Corral M, Garcia-Sanchez C, Pascual-Sedano B, Gironell A, Kulisevsky J (2010) Neuropsychological correlates of mild to severe hallucinations in Parkinson's disease. Mov Disord 25, 2785-2791. 
[14] Rahman S, Griffin HJ, Quinn NP, Jahanshahi M (2008) Quality of life in Parkinson's disease: The relative importance of the symptoms. Movement Disorders 23, 1428-1434.

[15] Yarnall AJ, Breen DP, Duncan GW, Khoo TK, Coleman SY, Firbank MJ, Nombela C, WinderRhodes S, Evans JR, Rowe JB (2014) Characterizing mild cognitive impairment in incident Parkinson disease The ICICLE-PD Study. Neurology 82, 308-316.

[16] Hughes AJ, Daniel SE, Kilford L, Lees AJ (1992) Accuracy of clinical diagnosis of idiopathic Parkinson's disease: a clinico-pathological study of 100 cases. Journal of Neurology, Neurosurgery \&amp; Psychiatry 55, 181-184.

[17] Emre M, Aarsland D, Brown R, Burn DJ, Duyckaerts C, Mizuno Y, Broe GA, Cummings J, Dickson DW, Gauthier S, Goldman J, Goetz C, Korczyn A, Lees A, Levy R, Litvan I, McKeith I, Olanow W, Poewe W, Quinn N, Sampaio C, Tolosa E, Dubois B (2007) Clinical diagnostic criteria for dementia associated with Parkinson's disease. Movement Disorders 22, 16891707.

[18] Tomlinson CL, Stowe R, Patel S, Rick C, Gray R, Clarke CE (2010) Systematic review of levodopa dose equivalency reporting in Parkinson's disease. Mov Disord 25, 2649-2653.

[19] Goetz CG, Tilley BC, Shaftman SR, Stebbins GT, Fahn S, Martinez-Martin P, Poewe W, Sampaio C, Stern MB, Dodel R, Dubois B, Holloway R, Jankovic J, Kulisevsky J, Lang AE, Lees A, Leurgans S, LeWitt PA, Nyenhuis D, Olanow CW, Rascol O, Schrag A, Teresi JA, van Hilten JJ, LaPelle N (2008) Movement Disorder Society-sponsored revision of the Unified Parkinson's Disease Rating Scale (MDS-UPDRS): Scale presentation and clinimetric testing results. Movement Disorders 23, 2129-2170.

[20] Hoehn MM, Yahr MD (1967) Parkinsonism: onset, progression and mortality. Neurology.

[21] Stebbins GT, Goetz CG, Burn DJ, Jankovic J, Khoo TK, Tilley BC (2013) How to identify tremor dominant and postural instability/gait difficulty groups with the movement disorder society unified Parkinson's disease rating scale: comparison with the unified Parkinson's disease rating scale. Movement Disorders 28, 668-670.

[22] Yesavage JA, Brink TL, Rose TL, Lum O, Huang V, Adey M, Leirer VO (1982) Development and validation of a geriatric depression screening scale: a preliminary report. J Psychiatr Res 17, 37-49.

[23] Razmy A, Lang AE, Shapiro CM (2004) Predictors of impaired daytime sleep and wakefulness in patients with parkinson disease treated with older (ergot) vs newer (nonergot) dopamine agonists. Archives of Neurology 61, 97-102.

[24] Buysse DJ, Reynolds CF, Monk TH, Berman SR, Kupfer DJ (1989) The Pittsburgh Sleep Quality Index: a new instrument for psychiatric practice and research. Psychiatry research 28, 193213.

[25] Peto V, Jenkinson C, Fitzpatrick R (1998) PDQ-39: a review of the development, validation and application of a Parkinson's disease quality of life questionnaire and its associated measures. Journal of Neurology 245, S10-S14.

[26] Folstein MF, Folstein SE, McHugh PR (1975) "Mini-mental state". A practical method for grading the cognitive state of patients for the clinician. J Psychiatr Res 12, 189-198. 
[27] Nasreddine ZS, Phillips NA, Bedirian V, Charbonneau S, Whitehead V, Collin I, Cummings JL, Chertkow H (2005) The Montreal Cognitive Assessment, MoCA: a brief screening tool for mild cognitive impairment. J Am Geriatr Soc 53, 695-699.

[28] Litvan I, Goldman JG, Tröster AI, Schmand BA, Weintraub D, Petersen RC, Mollenhauer B, Adler CH, Marder K, Williams-Gray CH (2012) Diagnostic criteria for mild cognitive impairment in Parkinson's disease: Movement Disorder Society Task Force guidelines. Movement Disorders 27, 349-356.

[29] Lawson RA, Yarnall AJ, Duncan GW, Khoo TK, Breen DP, Barker RA, Collerton D, Taylor JP, Burn DJ (2014) Severity of mild cognitive impairment in early Parkinson's disease contributes to poorer quality of life. Parkinsonism Relat Disord 20, 1071-1075.

[30] Mosimann UP, Collerton D, Dudley R, Meyer TD, Graham G, Dean JL, Bearn D, Killen A, Dickinson L, Clarke MP, McKeith IG (2008) A semi-structured interview to assess visual hallucinations in older people. International Journal of Geriatric Psychiatry 23, 712-718.

[31] Cubo E, Rojo A, Ramos S, Quintana S, Gonzalez M, Kompoliti K, Aguilar M (2002) The importance of educational and psychological factors in Parkinson's disease quality of life. European Journal of Neurology 9, 589-593.

[32] Schrag A (2006) Quality of life and depression in Parkinson's disease. Journal of the neurological sciences 248, 151-157.

[33] Marras C, Rochon P, Lang AE (2002) Predicting motor decline and disability in Parkinson disease: a systematic review. Archives of neurology 59, 1724-1728.

[34] Khoo TK, Yarnall AJ, Duncan GW, Coleman S, O'Brien JT, Brooks DJ, Barker RA, Burn DJ (2013) The spectrum of nonmotor symptoms in early Parkinson disease. Neurology 80, 276-281.

[35] Hobson P, Meara J (2004) Risk and incidence of dementia in a cohort of older subjects with Parkinson's disease in the United Kingdom. Movement Disorders 19, 1043-1049.

[36] McKinlay A, Grace RC, Dalrymple-Alford JC, Anderson T, Fink J, Roger D (2008) A profile of neuropsychiatric problems and their relationship to quality of life for Parkinson's disease patients without dementia. Parkinsonism \& Related Disorders 14, 37-42.

[37] Goetz CG, Stebbins GT (1995) Mortality and hallucinations in nursing home patients with advanced Parkinson's disease. Neurology 45, 669-671. 
Table 1. Demographic and clinical data

\begin{tabular}{lc}
\hline & $\begin{array}{c}\text { Participant } \\
\text { characteristics } \\
(\mathbf{n}=\mathbf{1 5 4})\end{array}$ \\
\hline Sex: male & $\mathbf{n}$ (\%) \\
Dopaminergic medication & $100(64.9)$ \\
PD-MCI & $135(87.7)$ \\
\hline & $68(41.2)$ \\
\hline Age & Mean \pm SD \\
Years of education & $66.4 \pm 10.4$ \\
Duration of PD (months) & $12.8 \pm 3.8$ \\
LEDD & $6.0 \pm 4.6$ \\
Hoehn and Yahr & $178.1 \pm 148.2$ \\
MoCA & $2.0 \pm 0.7$ \\
MMSE & $25.2 \pm 3.7$ \\
GDS-15 & $28.6 \pm 13.1$ \\
MDS-UPDRS part III & $2.8 \pm 2.5$ \\
ESS & $26.9 \pm 12.1$ \\
PDQ-39 & $6.2 \pm 4.5$ \\
PSQI & $18.7 \pm 14.4$ \\
\hline
\end{tabular}

MoCA = Montreal Cognitive Assessment, MMSE = Mini-Mental State Examination, MDS-UPDRS = Movement Disorder Society - Unified Parkinson's disease rating scale, LEDD = Levodopa equivalent daily dose, GDS-15 = 15 item Geriatric Depression Scale , PSQI - Pittsburgh Sleep Quality Index global score , ESS = Epworth Sleepiness Scale , PDQ-39 = Parkinson's Disease Questionnaire summary index.

$\mathrm{N}=15$ did not complete the MoCA 
Table 2: Clinical characteristics of newly diagnosed participants with Parkinson's disease with and without recurrent visual hallucinations

\begin{tabular}{|c|c|c|c|c|}
\hline & $\begin{array}{l}\text { Participants with } \\
\text { recurrent visual } \\
\text { hallucinations } \\
(n=34)\end{array}$ & $\begin{array}{l}\text { Participants without } \\
\text { recurrent visual } \\
\text { hallucinations } \\
(n=120)\end{array}$ & $t / \chi^{2} / Z$ & $\mathbf{P}$ \\
\hline Age (years) & $65.7 \pm 9.2$ & $66.6 \pm 10.7$ & 0.39 & $0.696^{t}$ \\
\hline Sex: male $n(\%)$ & $27(79.4)$ & $73(60.8)$ & 4.02 & $0.045^{x}$ \\
\hline PD-MCI n(\%) & $16(47.1)$ & $49(40.8)$ & 0.42 & $0.516^{x}$ \\
\hline Education (years) & $12.2 \pm 3.6$ & $12.9 \pm 3.9$ & -1.28 & 0.199 \\
\hline Disease duration (months) & $6.2 \pm 4.5$ & $6.0 \pm 4.6$ & -0.49 & 0.623 \\
\hline Motor Phenotype n (\%) & & & 5.53 & $0.063^{x}$ \\
\hline PIGD & $21(61.8)$ & $48(40.0)$ & & \\
\hline Indeterminate & $5(14.7)$ & $20(16.7)$ & & \\
\hline Tremor dominant & $8(23.5)$ & $52(43.3)$ & & \\
\hline MoCA & $23.8 \pm 3.9$ & $25.5 \pm 3.5$ & -2.24 & 0.025 \\
\hline MMSE & $28.2 \pm 1.4$ & $28.8 \pm 1.3$ & -2.26 & 0.024 \\
\hline MDS-UPDRS Part II & $12.3 \pm 5.4$ & $10.4 \pm 5.8$ & -1.75 & 0.081 \\
\hline MDS-UPDRS Part III & $28.3 \pm 12.0$ & $26.5 \pm 12.1$ & -0.74 & $0.459^{t}$ \\
\hline Hoehn and Yahr & $2.0 \pm 0.7$ & $2.0 \pm 0.7$ & -0.24 & 0.815 \\
\hline LEDD (mg/day) & $186.9 \pm 149.1$ & $175.5 \pm 148.4$ & -0.70 & 0.485 \\
\hline GDS-15 & $3.9 \pm 3.1$ & $2.5 \pm 2.3$ & -2.43 & 0.015 \\
\hline PSQI & $7.2 \pm 4.7$ & $6.0 \pm 3.7$ & -1.13 & 0.260 \\
\hline ESS & $8.8 \pm 5.0$ & $5.4 \pm 4.1$ & -3.52 & $<0.001$ \\
\hline PDQ-39 & $26.7 \pm 17.0$ & $16.4 \pm 12.7$ & -3.28 & 0.001 \\
\hline
\end{tabular}

Data presented as mean \pm SD unless otherwise stated $X=$ chi squared, $t=$ independent samples $t$ test, remainder of tests were MannWhitney $U$ test. Significant results highlighted in bold $(p<0.05)$

PD-MCI = Mild Cognitive Impairment at 1.5SD, PIGD = Postural Instability Gait Difficulty, MoCA = Montreal Cognitive Assessment, MMSE = Mini-Mental State Examination, MDS-UPDRS = Movement Disorder Society - Unified Parkinson's disease rating scale, LEDD = Levodopa equivalent daily dose, GDS-15 = 15 item Geriatric Depression Scale , PSQI - Pittsburgh Sleep Quality Index global score , ESS = Epworth Sleepiness Scale , PDQ-39 = Parkinson's Disease Questionnaire summary index.

$\mathrm{N}=15$ did not complete the MoCA 
Table 3. Clinical characteristics of newly diagnosed participants with Parkinson's disease and differing severities of visual hallucinations experienced

\begin{tabular}{|c|c|c|c|c|c|c|}
\hline & $\begin{array}{l}\text { Minor VHs } \\
(\mathrm{N}=10)\end{array}$ & Simple VHs (N=9) & Illusions $(\mathrm{N}=4)$ & $\begin{array}{l}\text { Complex VHs } \\
(\mathrm{N}=11)\end{array}$ & $\mathrm{X}^{2}$ & $\mathbf{P}$ \\
\hline Age (years) & $66.7 \pm 11.4$ & $66.9 \pm 9.0$ & $69.1 \pm 11.9$ & $67.4 \pm 5.5$ & 2.2 & 0.537 \\
\hline Sex: male n (\%) & $7(70.0)$ & $7(77.8)$ & $3(75.0)$ & 10 (90.9) & 5.1 & $0.278^{x}$ \\
\hline PD-MCI n (\%) & $6(60.0)$ & $4(44.4)$ & $2(50.0)$ & $4(36.4)$ & 1.7 & $0.797^{x}$ \\
\hline Education (years) & $11.3 \pm 3.3$ & $13.7 \pm 3.7$ & $11.0 \pm 0.8$ & $12.2 \pm 4.2$ & 2.7 & 0.448 \\
\hline Disease duration (months) & $4.8 \pm 3.3$ & $6.6 \pm 7.4$ & $7.9 \pm 4.2$ & $6.8 \pm 2.1$ & 3.7 & 0.291 \\
\hline Motor phenotype n (\%) & & & & & 11.2 & $0.191^{x}$ \\
\hline PIGD & $9(90.0)$ & $4(44.4)$ & $2(50.0)$ & $6(54.5)$ & & \\
\hline Indeterminate & $1(10.0)$ & $2(22.2)$ & $1(25.0)$ & $1(9.1)$ & & \\
\hline Tremor Dominant & $0(0.0)$ & $3(33.3)$ & $1(25.0)$ & $4(36.4)$ & & \\
\hline MoCA & $24.1 \pm 4.1$ & $23.4 \pm 4.5$ & $23.3 \pm 2.1$ & $24.0 \pm 4.1$ & 0.3 & 0.953 \\
\hline MMSE & $28.1 \pm 1.5$ & $28.3 \pm 1.3$ & $28.5 \pm 1.0$ & $28.1 \pm 1.6$ & 0.3 & 0.968 \\
\hline MDS-UPDRS Part II & $14.3 \pm 6.2$ & $10.2 \pm 4.3$ & $12.5 \pm 5.9$ & $12.2 \pm 5.3$ & 1.4 & 0.696 \\
\hline MDS-UPDRS Part III & $27.2 \pm 12.2$ & $26.8 \pm 12.0$ & $34.0 \pm 11.0$ & $28.4 \pm 13.2$ & 1.5 & 0.686 \\
\hline Hoehn and Yahr & $2.2 \pm 0.8$ & $1.8 \pm 0.7$ & $2.5 \pm 0.6$ & $1.8 \pm 0.6$ & 4.6 & 0.208 \\
\hline LEDD (mg/day) & $173.0 \pm 74.5$ & $206.7 \pm 186.5$ & $130.0 \pm 124.9$ & $204.1 \pm 183.6$ & 0.8 & 0.826 \\
\hline GDS-15 & $4.2 \pm 3.4$ & $3.8 \pm 4.1$ & $4.5 \pm 1.0$ & $3.4 \pm 2.7$ & 1.8 & 0.610 \\
\hline PSQI & $6.9 \pm 5.1$ & $6.7 \pm 5.4$ & $7.3 \pm 3.6$ & $7.8 \pm 4.6$ & 0.7 & 0.883 \\
\hline ESS & $9.3 \pm 6.7$ & $7.8 \pm 4.2$ & $6.8 \pm 3.7$ & $9.8 \pm 4.5$ & 2.2 & 0.542 \\
\hline PDQ-39 & $28.0 \pm 18.6$ & $23.7 \pm 17.0$ & $31.8 \pm 18.3$ & $26.1 \pm 17.2$ & 0.9 & 0.832 \\
\hline
\end{tabular}

Data presented as mean \pm SD unless otherwise stated $\mathrm{X}=$ chi squared, $\mathrm{t}=$ independent samples $\mathrm{t}$ test, remainder of tests were Mann-Whitney $\mathrm{U}$ test. Post hoc Bonferroni correction for group comparison at $\mathrm{p}<$ 0.008 .

PD-MCI = Mild cognitive impairment at 1.5 SD, PIGD = Postural Instability Gait Difficulty, MoCA = Montreal Cognitive Assessment, MMSE = Mini-Mental State Examination, MDS-UPDRS = Movement Disorder Society Unified Parkinson's disease rating scale, LEDD = Levodopa equivalent daily dose, GDS-15 = 15 item Geriatric Depression Scale, PSQI - Pittsburgh Sleep Quality Index global score , ESS = Epworth Sleepiness Scale, PDQ-39 = Parkinson's Disease Questionnaire summary index, VHs = visual hallucinations. Data presented as mean \pm SD unless otherwise stated. $\mathrm{N}=4$ did not complete the MoCA. 
Table 2: Regression coefficients and model fit of predictors of MoCA, PDQ-39 and MDS-UPDRS part III scores.

\begin{tabular}{|c|c|c|c|c|c|c|}
\hline & & \multirow{3}{*}{$\mathbf{t}$} & \multirow{3}{*}{$\mathbf{p}$} & \multirow{3}{*}{$\beta$} & \multicolumn{2}{|c|}{$95 \% \mathrm{Cl}$ for $\beta$} \\
\hline & & & & & Lower & Upper \\
\hline & & & & & Bound & Bound \\
\hline \multirow[t]{4}{*}{ MoCA } & Initial model ${ }^{\mathrm{a}}$ & & & & & \\
\hline & Age & -4.1 & $<0.001$ & -0.3 & -0.2 & -0.1 \\
\hline & Education & 3.0 & 0.003 & 0.2 & 0.1 & 0.4 \\
\hline & Initial model + Recurrent $\mathrm{VHs}^{\mathrm{b}}$ & -2.6 & 0.010 & -0.2 & -3.0 & -0.4 \\
\hline \multirow[t]{6}{*}{$P D Q-39$} & Initial model $^{\mathrm{c}}$ & & & & & \\
\hline & GDS-15 & 9.0 & $<0.001$ & 0.5 & 2.3 & 3.6 \\
\hline & MDS-UPDRS part III & 4.5 & $<0.001$ & 0.3 & 0.2 & 0.5 \\
\hline & Age & -3.6 & $<0.001$ & -0.2 & -0.5 & -0.1 \\
\hline & Education & -2.4 & 0.020 & -0.1 & -1.0 & -0.1 \\
\hline & Initial model + Recurrent $\mathrm{VHs}^{\mathrm{d}}$ & 2.8 & 0.006 & 0.2 & 1.5 & 9.3 \\
\hline \multirow[t]{4}{*}{ MDS-UPDRS Part III } & Initial model $^{\mathrm{e}}$ & & & & & \\
\hline & GDS-15 & 3.5 & 0.001 & 0.3 & 0.6 & 2.0 \\
\hline & PD-MCl & 2.5 & 0.012 & 0.2 & 1.0 & 8.5 \\
\hline & Initial model + Recurrent VHs ${ }^{f}$ & -0.1 & 0.906 & 0.0 & -4.8 & 4.2 \\
\hline
\end{tabular}

a: $R=0.477, R^{2}=0.227$, adjusted $R^{2}=0.216, F=20.0, p<0.001, S E=3.2$

$b: R=0.514, R^{2}=0.264$, adjusted $R^{2}=0.248, F=16.2, p<0.001, S E=3.2, \Delta R^{2}=0.037$

c: $R=0.725, R^{2}=0.526$, adjusted $R^{2}=0.514, F=41.4, p<0.001, S E=10.0$

$d: R=0.741, R^{2}=0.549$, adjusted $R^{2}=0.534, F=36.1, p<0.001, S E=9.8, \Delta R^{2}=0.023$

e: $R=0.358, R^{2}=0.128$, adjusted $R^{2}=0.116, F=11.1, p<0.001, S E=11.4$

$f: R=0.358, R^{2}=0.128$, adjusted $R^{2}=0.111, F=7.3, p<0.001, S E=11.4, \Delta R^{2}=0.000$

$\mathrm{Cl}=$ confidence intervals, MoCA = Montreal Cognitive Assessment, $\mathrm{VHs}=$ visual hallucinations, MDS-UPDRS = Movement Disorder Society Unified Parkinson's disease rating scale, GDS-15 = 15 item Geriatric Depression Scale, PDQ-39 = Parkinson's Disease Questionnaire summary index, $P D-M C I=$ Mild Cognitive Impairment applying cut-off of $1.5 S D$ below normative values, Significant results highlighted in bold. 


\section{Supplementary Table 3. Summary of visual hallucination classification}

\begin{tabular}{ll}
\hline $\begin{array}{l}\text { Classification of Visual } \\
\text { Hallucination }\end{array}$ & \multicolumn{1}{c}{ Description } \\
\hline Complex Hallucination & $\begin{array}{l}\text { Formed visual hallucination such as people, animals, objects, devils } \\
\text { etc. }\end{array}$ \\
\hline Allusion & $\begin{array}{l}\text { A misperception in vision e.g. wallpaper patterns move, perceiving } \\
\text { clothing as an animal or person. }\end{array}$ \\
\hline $\begin{array}{l}\text { Simple Hallucination - } \\
\text { without migraine }\end{array}$ & $\begin{array}{l}\text { Unformed visual hallucinations such as lights, spots, colours, } \\
\text { shadows. }\end{array}$ \\
\hline Simple Hallucination - & Unformed visual hallucinations as described above that occur \\
in context of migraine & during a migraine. \\
\hline Minor Hallucination - & The feeling of a person, being or thing nearby or out of view. \\
Presence & \\
\hline Minor Hallucination - & The feeling or vision of an animal, shadow or person passing by. \\
Passage & Generally spots which move with the individuals eyes. \\
\hline Floater &
\end{tabular}

